\title{
2D size, position and shape definition of defects by B-scan image analysis
}

\author{
Michele Scafidi, Donatella Cerniglia, Tommaso Ingrassia \\ Università degli Studi di Palermo, Dipartimento di Ingegneria Chimica, Gestionale, Informatica, Meccanica - 90128 Palermo, \\ Italy \\ tommaso.ingrassia@unipa.it
}

\begin{abstract}
The non-destructive evaluation of defects by automatic procedures is of great importance for structural components. Thanks to the developments of the non-contact ultrasonic techniques, the automation of the inspections is gaining a progressively important role. In this work, an automatic inspection technique for the evaluation of defects by the analysis of B-scan images obtained by a laser ultrasonic system is presented. The data are extracted directly from a B-scan map obtained for a panel with internal defects, and are used to build an image of the cross section of the panel. The proposed automatic procedure allows the definition of size, position and shape of defects in panels of known thickness.
\end{abstract}

KEYWORDS. NDE; laser UT system; B-scan image analysis; defect size definition; 2D defect shape definition.

\section{INTRODUCTION}

$\mathrm{T}$ he laser Ultrasonic Testing (UT) systems are becoming more common among the Non-Destructive Evaluation (NDE) techniques thanks to the possibility to carry out non-contact inspections [1-3]. One of the most important advantage of this technique is related to the use of high frequency ultrasonic waves, that allow the detection of defects with very fine spatial resolution. Moreover, laser UT systems can be effectively used for remote inspections with no contact conditions influence and, if proper delivery optics are used to guide the laser beam, they can operate also in hostile environments.

Since the propagation mechanism of the ultrasonic waves is not influenced by the angle of incidence of the laser beam on the material surface, this kind of system can be used to inspect parts where access is limited. The laser beam, in fact, can be directed to the surface with high angles off axis.

The presence of defects, corners and curved surfaces, modifies the waves propagation, causing reflection and mode conversion. The waves resulting from different sources (i.e. reflected or converted waves) can interfere each other, generating very complex patterns. For this reason, the inspection of complex structures by means of laser UT systems can become extremely hard. Nevertheless, by knowing the analytical models of the waves propagation in solid structures, the experimental layout can be designed to optimize the results post-processing analysis.

With this purpose, laser UT systems can be used to automate the scanning procedure and to make a rapid acquisition of the ultrasonic data by creating B-scan maps in real time [1]. The analysis of the B-scan image allows determining in an automated way the presence of defects in the tested component as well as the characteristics of the defects. A technique of particular importance in the analysis of defects in plates is the Time Of Flight Diffraction (TOFD) [4-9]. This technique allows to determine the presence of cracks in the material and to determine the position and length of the crack even in 
components of complex geometry [10-13]. To facilitate the automation of the analysis, TOFD B-scan maps with selected wave types can be obtained by optimizing the layout of the laser system.

The aim of this work is the automatic definition of size, shape and position of the defects in plates of known thickness. A virtual image of the section of the analyzed component is created by an algorithm that locates the areas with defects. A second algorithm allows determining also the boundary shape of the defect.

\section{ULTRASONIC SYSTEM AND LASER LAYOUT DESCRIPTION}

he laser system used in this work, shown in Fig. 1, consists of:

- an IR Nd:YAG pulsed laser for the ultrasonic wave generation;

- a CW laser interferometer as receiver system for the out-of-plane displacements measurement;

- a motorized linear micro-slide;

- a system for data acquisition and processing.

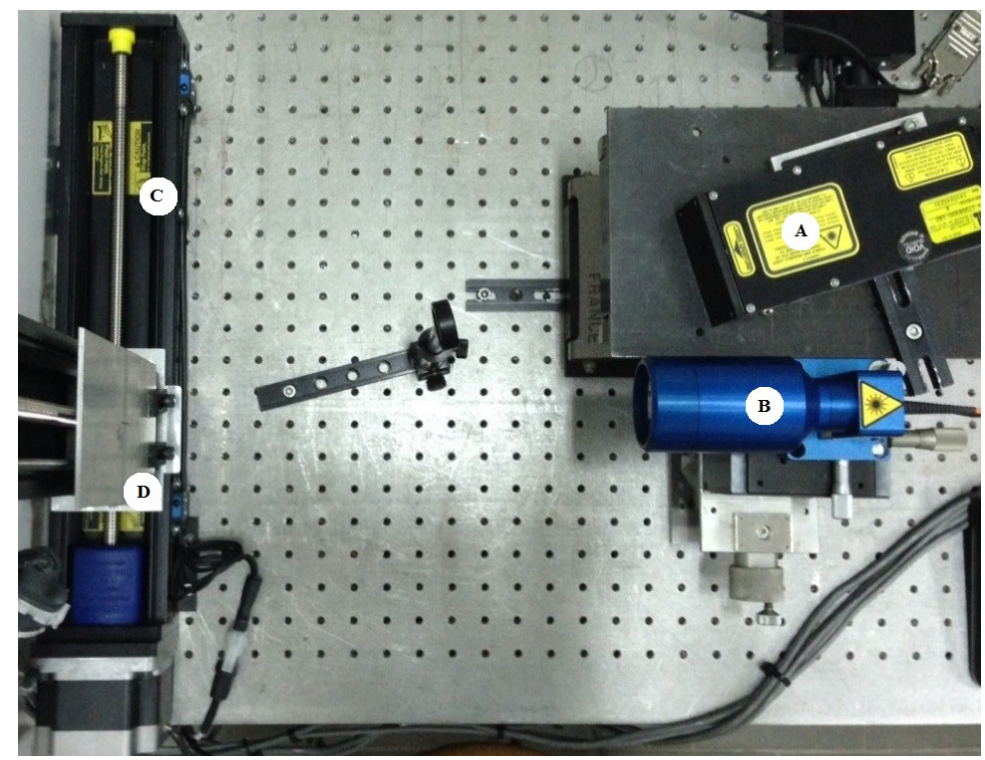

Figure 1: Laser system: (A) IR Nd:YAG pulsed laser for ultrasonic wave generation; (B) CW laser interferometer receiver; (C) motorized linear micro-slide; (D) sample.

In the application here considered, the wave generation and detection are made on the same side of a panel of thickness $T$ at a defined distance $D$ (see Fig. 2). The laser source generates longitudinal, shear and surface waves in the ablation regime whose angular dependence is reported in ref. [4].

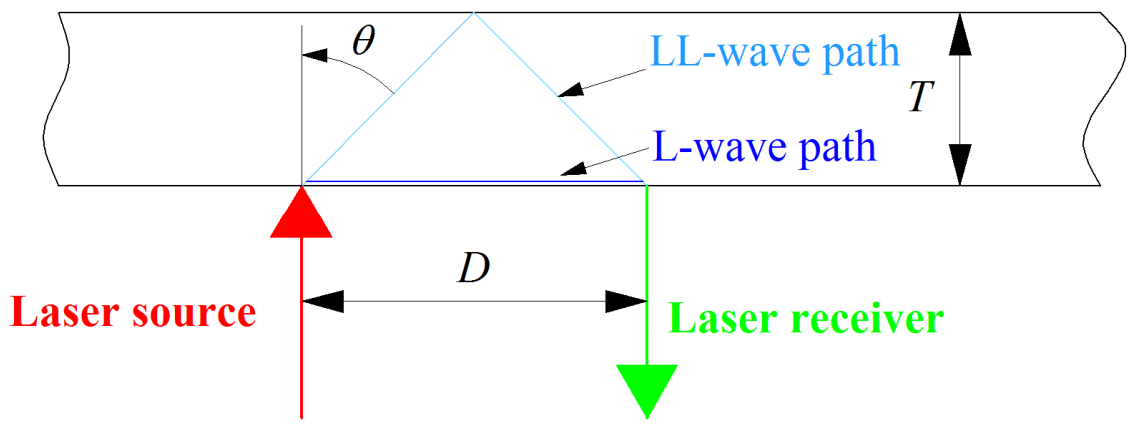

Figure 2: Cross-section of a plate with indication of laser source, laser receiver and wave paths. 
As shown in Fig. 2, the surface skimming longitudinal wave, L-wave, travels just below the surface of the plate; the longitudinal back-wall wave, LL-wave, reflected by the opposite plate surface in accordance with the Snell's law, travels with an orientation $\theta$ that depends on the distance $D$.

Being the longitudinal wave velocity $v_{L}$ about twice than the shear and surface wave velocities [14] and considering that the L-wave path (length $D$ ) is the shortest between the generation point and the receiver point, the L-wave is the first that reaches the receiver. If the LL-wave travels along a path with length $D_{L L}=2 \mathrm{D}$, the surface wave, the shear wave and the LL-wave reach the receiver at about the same time. This condition occurs when the triangle of the wave path is equilateral $\left(\theta=30^{\circ}\right)$ but, in this case, it is impossible to identify the three different waves apart. Choosing a propagation angle $\theta=45^{\circ}$, instead, the distance between the two lasers is $D=2 T$ and the total length of the LL-wave path is $D_{L L}=2 T \sqrt{ } 2=2.83 T \cong 1.4 D$. This choice ensures that the LL-wave can be entirely acquired, cutting off the surface wave and the shear wave from the time window. Furthermore, the intensity of the out-of-plane component of the LL-wave for $\theta=45^{\circ}$ is about $70 \%$ of the LL-wave intensity. In this way, in line with the purposes of this research, the distance $D$ and the time-window have been then optimized to visualize only the L-wave and the LL-wave.

In Fig. 3, a signal acquired by the receiver, in a $T=5 \mathrm{~mm}$ aluminum plate without defect and a source/receiver distance of $D=10 \mathrm{~mm}$, is shown.

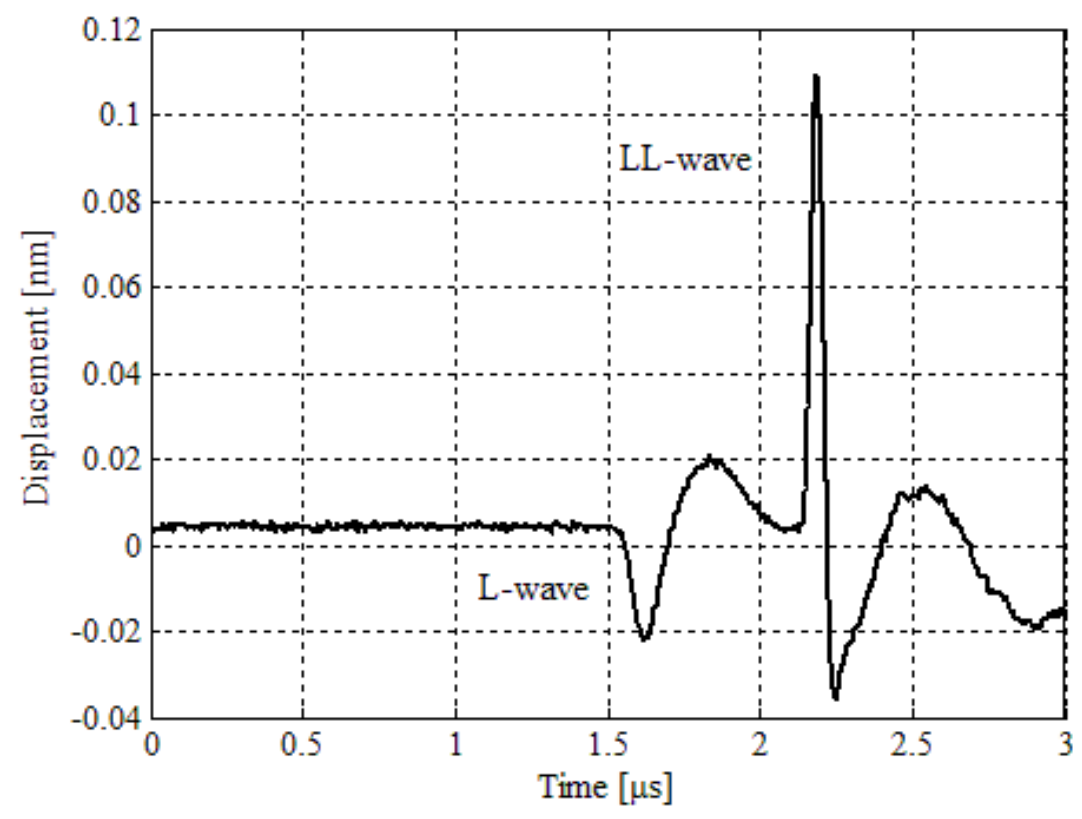

Figure 3: Typical signal of the longitudinal waves without defect.

In Fig. 4, the section of the analyzed plate with the laser layout scheme is shown.

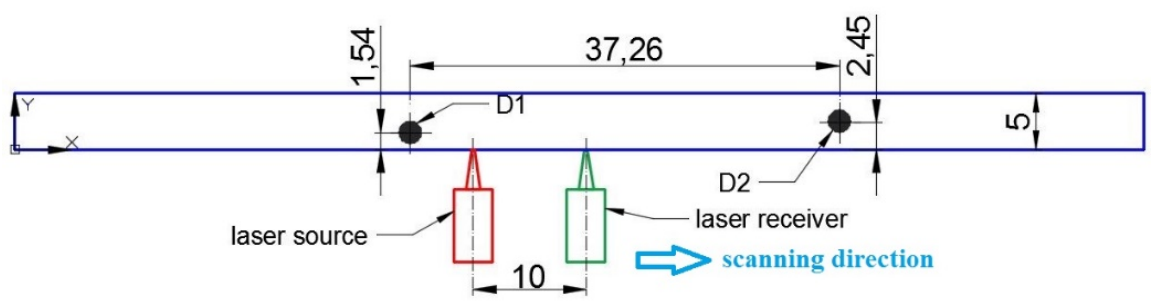

Figure 4: Section of the analyzed aluminum plate and laser layout (measures in $\mathrm{mm}$ ).

In the plate two circular holes, $D_{1}$ and $D_{2}$, were drilled. Their diameters are, respectively, $2.10 \mathrm{~mm}$ and $2.09 \mathrm{~mm}$. The distances of the defects centers from the scanning surface ( $y$-depth) are, respectively, $y_{1}=1.54 \mathrm{~mm}$ and $y_{2}=2.45 \mathrm{~mm}$. The distance, $\mathrm{L}$, between the centers of the two defects is $37.26 \mathrm{~mm}$. 
To analyze the plate, a B-scan map has been built by regularly shifting, along a $100 \mathrm{~mm}$ straight line, the laser system and storing the signal for each step. Fig. 5 shows the B-scan map obtained with the panel of Fig. 4. The laser source position defines the abscissa $x_{G}$ of the B-scan map. Two perturbations can be noted due to the presence of the defects.

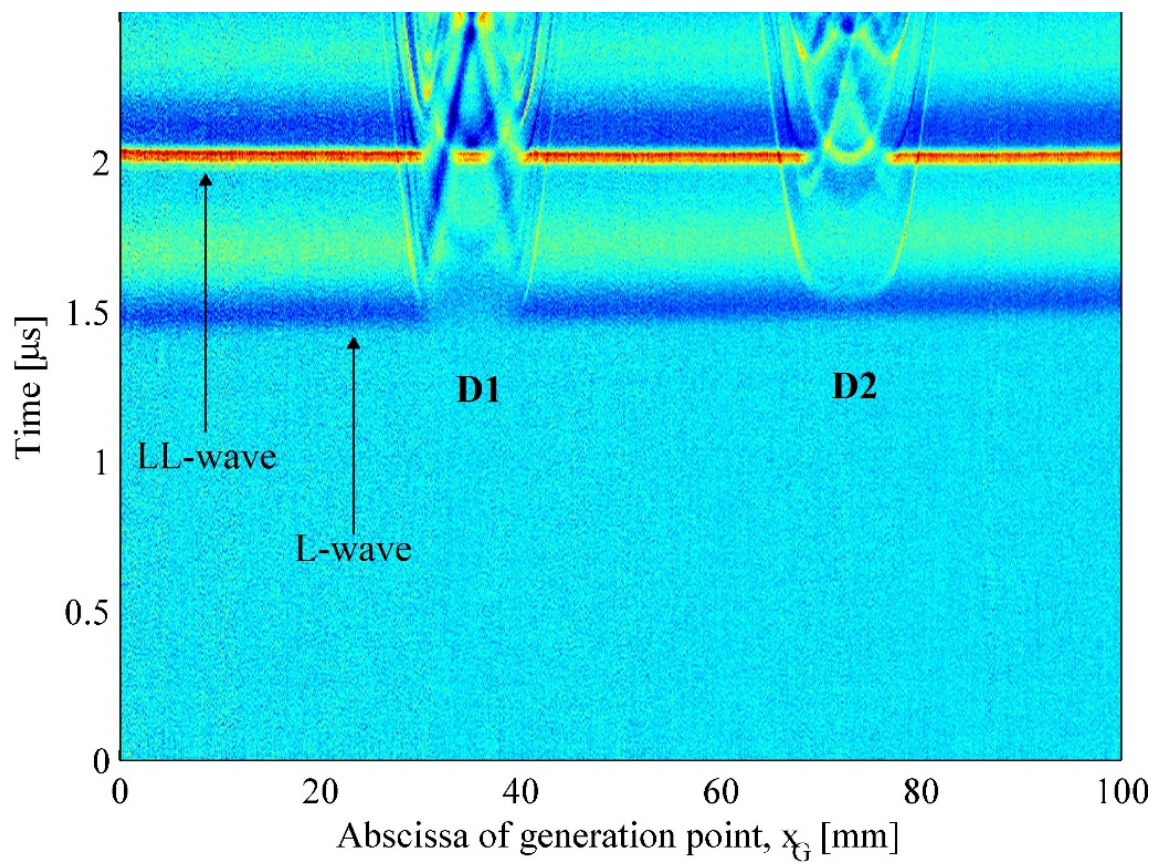

Figure 5: B-scan map obtained for the plate of fig. 4 with indication of the L-wave negative and LL-wave positive peaks.

The B-scan map is shown in false-colours to highlight the peak-to-peak amplitude of the signals.

Similarly to the case of the waves diffracted by the crack tip $[6,7,13]$, the perturbation shows a parabolic-like pattern in the B-scan. In general, the defect affects the shape of the perturbation then, analyzing this last, the defect characteristics can be determined. Unfortunately, if the presence of the perturbations on the B-scan map can clearly make in evidence the presence of defects, the definition of their size, position and shape requires a more complex analysis. In the next sections, the proposed procedures for defining the size, the position and the shape of the defects are described.

\section{B-SCAN ANALYSIS: SIZE AND POSITION DEFINITION}

$\mathrm{T}$

he LL-wave (red line in Fig. 5) is interrupted twice in correspondence of the defect D1. These interruptions are caused by the interposition of the defect along the LL-wave path, as shown in the Fig. 6. From the B-scan, it would seem that the defect interrupts also the L-wave. Actually, this effect is caused by the superposition of the longitudinal wave, L-wave, and the reflected wave due to the defect, LR-wave. In this case, in fact, due to the little depth (y) of the defect D1, the LL-wave and the LR-wave have similar path lengths.

A different case, shown in Fig. 7, is represented by the defect D2 that has a greater depth. The LL-wave is interrupted only one time but for a bigger length.

For the defect D1, the length of the interruptions depends on the defect size while for the defect D2, the length of the interruption depends also on the depth.

As general case, measuring the length of the interruptions of the LL-wave directly on the B-scan map, information about the dimension of the defect can be obtained, while information on its depth can be hardly extracted.

For a better definition of the size and position of the defect, the draft of an image of the section, considering the information extracted by the B-scan, is here proposed. This section image consists in drawing, for each scan step, a broken line describing the path of the LL-wave. These path-lines are drawn in a gray colour whose intensity is proportional to the amplitude of the corresponding acquired signal. Each pixel of the section image is obtained as intersection of the path-lines of two different steps. The biggest intensity of the (two) intersecting lines is assigned to the pixel. In this way, a bright pixel belongs to one not-interrupted path whereas a dark colored pixel is part of two 
interrupted paths. Therefore, defects cannot be in the bright zones of the section image, while they could be in the dark areas. In Fig. 8, the section image built with the data extracted from the B-scan of Fig. 5 is shown.

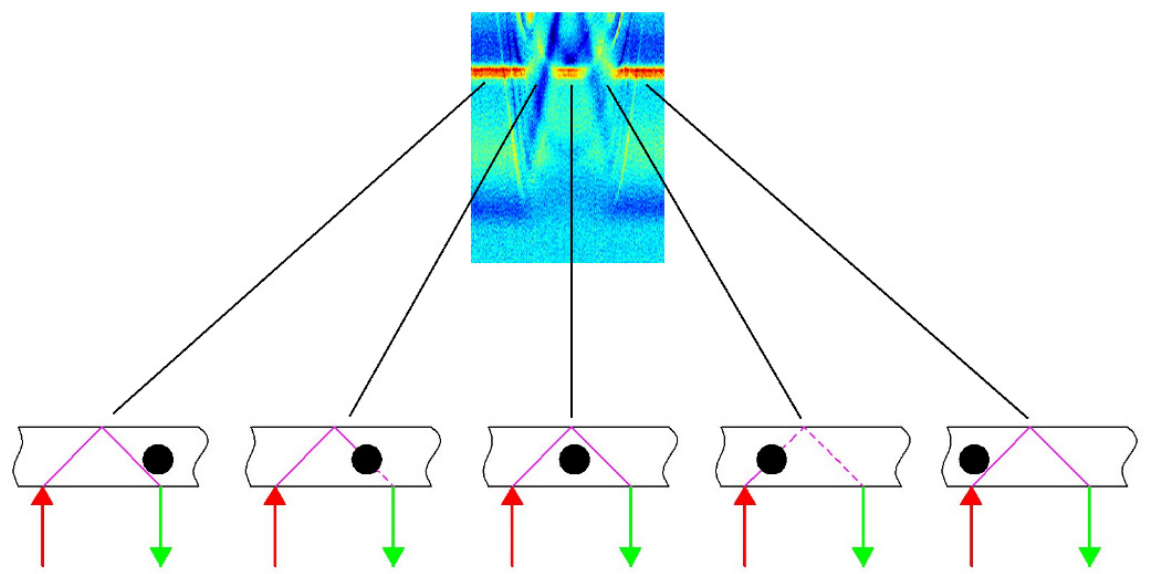

Figure 6: Interruption of the LL-wave due to the defect D1 and corresponding effects on the B-scan map.

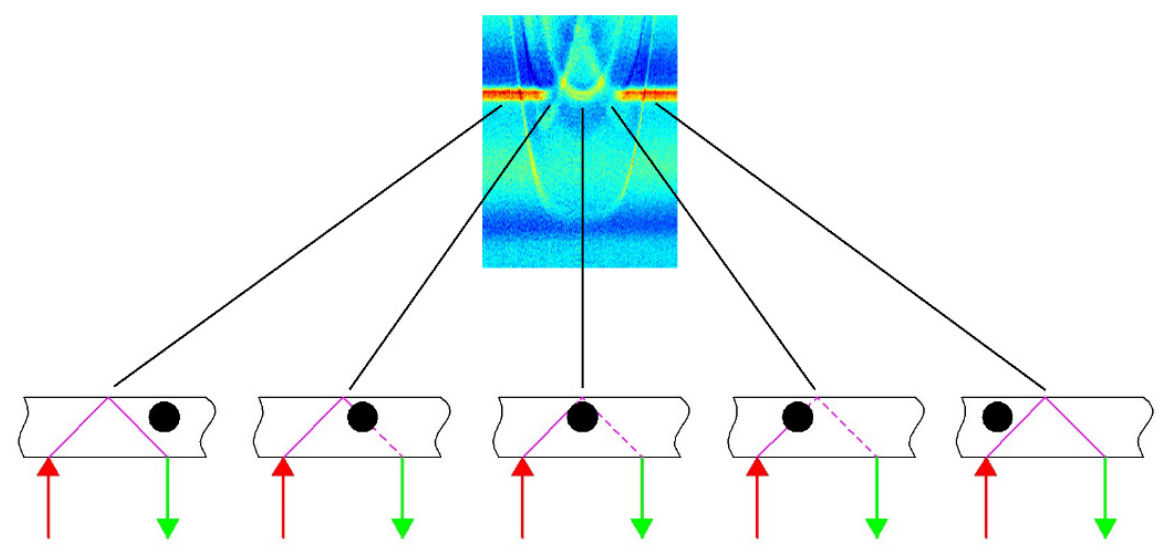

Figure 7: Interruption of the LL-wave due to the defect D2 and corresponding effects on the B-scan map.

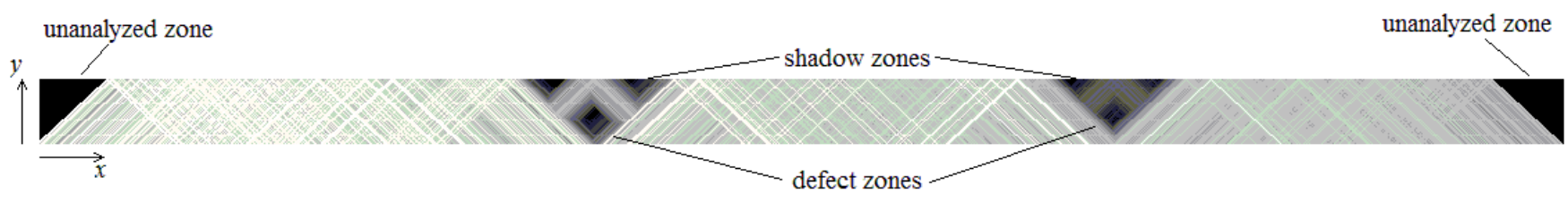

Figure 8: Section image built from the data of the B-scan of Fig. 5.

In Fig. 8, some "shadow" and unanalyzed zones are highlighted. In these last areas, no signal was acquired because the extreme parts of the plate have not been scanned. The shadow zones, instead, correspond to low-intensity signals. In general, in these areas the presence of defects cannot be excluded observing only the section image built with the described procedure. The shadow zones with no defects could be removed by performing the inspection also from the other side of the panel. For the purposes of this research, the shadow zones and the unanalyzed zones have not been considered.

To extract the information about the size and the position of the defect, a binarized section image has been built from the section image of Fig. 8 by a threshold procedure. To optimize the results, proper threshold values have been chosen depending on the defect characteristics. In particular, at this stage of the research, they have been defined knowing the value of the defect diameter. The best threshold values are, respectively, $40 \%$ and $35 \%$ of the maximum intensity for the defect D1 and D2. The binarized section images are shown in Fig. 9. 


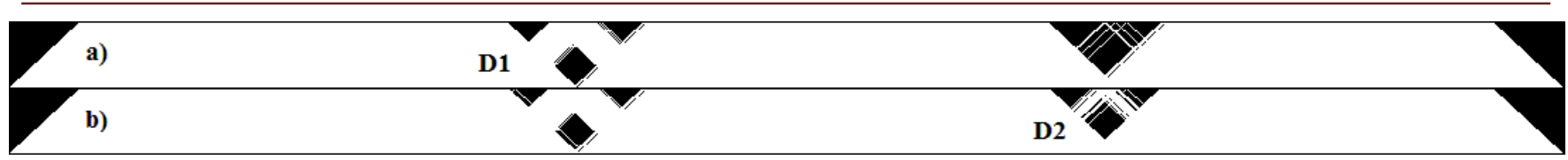

Figure 9: Binarized section images obtained with a threshold value of 40\% (a) for D1 and 35\% (b) for D2.

The analysis of the section images allows to determine the positions of the defects by identifying the center of gravity $(C G)$ of the dark zones. The procedure consists in calculating the coordinates of the CG by the following equations:

$$
\begin{aligned}
& x_{g}=\frac{\sum x_{i}}{N} \\
& y_{g}=\frac{\sum y_{i}}{N}
\end{aligned}
$$

where $x_{i}$ and $y_{i}$ are the coordinates of the generic dark pixel, while $N$ is the total number of pixels in the dark zones.

From the analysis of the section image, the $x$ coordinates of the center of the defects are $x_{1}=40.2 \mathrm{~mm}$ and $x_{2}=77.7 \mathrm{~mm}$, respectively for D1 and D2. Consequently, the calculated distance between the two defects is $L=37.5 \mathrm{~mm}$. This value is in a very good agreement with the real one, equal to $37.26 \mathrm{~mm}$, and the error is about $0.6 \%$.

The $y$ positions (depth) of the center of the defects result $y_{1}=1.62 \mathrm{~mm}$ for D1 and $y_{2}=2.32 \mathrm{~mm}$ for D2. The errors on the depth are, respectively, about $+5.2 \%$ and $-5.3 \%$.

\section{B-SCAN ANALYSIS: DEFECT SHAPE DEFINITION}

he shape of the defects has been determined by analyzing the B-scan map perturbations due to the defects (Fig. 5). Because of the regular shape of the drilled holes, the perturbation is not caused by the diffraction [4-9] but is due to the reflection of the longitudinal waves at the smoothed boundary of the hole. As shown in Fig. 10, for each scan step, a longitudinal wave (LR-wave), starting from the generation point, hits the defect and is reflected to the receiver point.

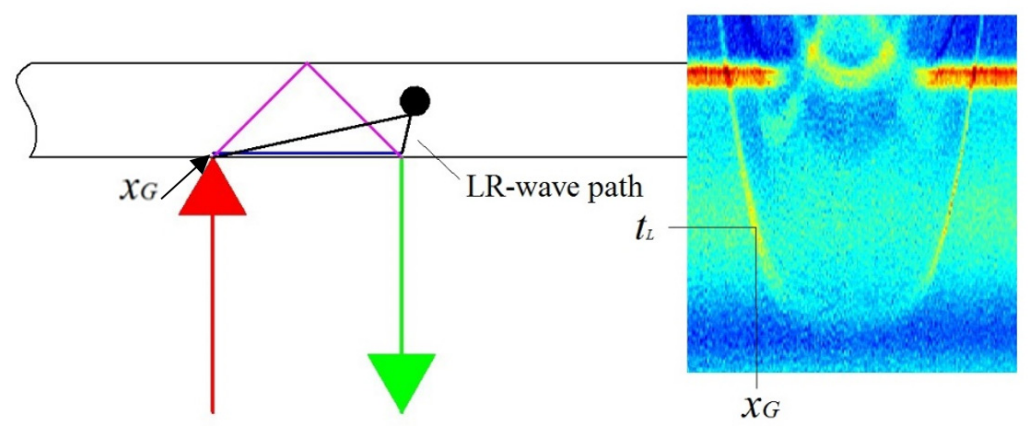

Figure 10: Path of a generic LR-Wave (left) and corresponding point in the B-scan image (right).

At each step, the laser receiver acquires the signal of the LR-wave reflected by the closer point of the defect. In this way, the bottom boundary of the perturbation on the B-scan map is defined. Extracting the coordinates $\mathrm{x}_{\mathrm{G}}$ of the perturbation boundary points from the B-scan, the time of arrival $t_{L}\left(x_{G}\right)$ of the LR-waves can be determined (fig. 10).

Known the wave velocity $v_{L}$ of the longitudinal wave, the length of the path $L_{L}\left(x_{G}\right)$ covered by the LR-wave can be determined by the following equation:

$$
L_{L}\left(x_{G}\right)=v_{L} \cdot t_{L}\left(x_{G}\right)
$$

As seen in Fig.10, the path of the LR-wave is composed of two parts: the first one goes from the generation point to the reflection point, the second one from this last point to the receiver. Since the initial direction of the LR-wave and the 
defect shape and position are unknown, the lengths of the two parts of the path cannot be determined separately. Under these conditions, all the points for which the sum of the distance from two fixed point (generation and receiver point) is $L_{L}\left(x_{G}\right)$ could be reflection points. In particular, all the points on the semi-ellipse with generation and receiver points as foci and with the sum of the distances from them equal to $L_{L}\left(x_{G}\right)$ could be reflection points. Since the generation and receiver points are on the $x$-axis (fig. 4), the generic equation of the ellipse having the possible reflection points is:

$$
\frac{\left(x-x_{c}\right)^{2}}{a^{2}}+\frac{y^{2}}{b^{2}}=1
$$

where $a$ and $b$ are, respectively, the major and minor semi-axes of the ellipse, whose values, for the analyzed case, are:

$$
\begin{aligned}
& a=\frac{L_{L}\left(x_{G}\right)}{2} \\
& b=\sqrt{\left(\frac{L_{L}\left(x_{G}\right)}{2}\right)^{2}-\left(\frac{D}{2}\right)^{2}}
\end{aligned}
$$

and $x_{c}$ is the abscissa of the center of the ellipse (central point between the two foci at $D$ distance), that is linked to the generation point by the following relationship:

$$
x_{c}=x_{G}+\frac{D}{2}
$$

Finally, for each scan step, knowing $x_{G}$ and calculating $L_{L}\left(x_{G}\right)$, the ellipse passing through the reflecting point of the defect can be drawn. The envelope of all the ellipses determines the shape of the defect.

Fig. 11 shows, for the defect D1, the envelope of the ellipses superimposed to the circular profile of the drilled hole. A very good agreement is found in the matching of the experimental and real data.

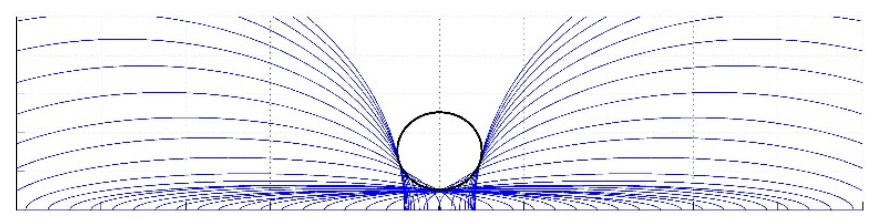

Figure 11: Envelope of the ellipses (blue lines) drawn for the defect D1 and defect contour (black circle).

The envelope of the ellipses shown in Fig. 8 determines only one half of the defect profile. The other part of the profile can be determined by applying the same algorithm to the experimental data coming from an inspection performed in the opposite side of the panel.

\section{CONCLUSIONS}

A new procedure of analysis of the B-scan image obtained by laser UT for NDE has been presented in this paper. It has been shown that, using an appropriate lay-out optimized to detect the longitudinal wave reflected by the opposite side of the panel, it is possible to define the main characteristics of the defects. In particular, the size, the position and the shape of the defect can be determined.

The main dimensions and the position of the defect can be defined analyzing some section images built from the B-scan maps. These section images, if suitably filtered, allow to calculate the coordinates of the defect center. 
The analysis of the perturbation in the B-scan, due to the presence of the defects, allows determining the shape of the defect. The definition of the shape of the defect is achieved by means of the envelope of the ellipses drawn on the image section, by measuring the time of arrival of the wave reflected by the defect from the B-scan.

Although the research requires a calibration procedure and the automation of some parts of the analysis, the results obtained are in good agreement with the characteristics of the defects created into the aluminum panel used as case study. The next steps of the research are the reduction of the effects of the shadow zones, the improvement and the automation of the procedures to extract position, size and shape of the defects.

\section{REFERENCES}

[1] Cerniglia, D., Scafidi, M., Pantano, A., Rudlin, J., Inspection of additive-manufactured layered components, Ultrasonics, 62 (2015) 292-298.

[2] Rudlin, J., Cerniglia, D., Scafidi, M., Inspection of laser powder deposited layers, Proceedings of 52nd Annual Conference of the British Institute of Non-Destructive Testing (2013), BINDT 2013 - Telford (UK), (2013).

[3] Cerniglia, D., Djordjevic, B.B., Ultrasonic detection by photo-EMF sensor and by wideband air-coupled transducer, Research in Nondestructive Evaluation, 15 (2004) 111-117.

[4] Scruby, C.B., Drain, L., Laser Ultrasonics: Techniques and Applications, Adam Hilger, Bristol, (1990).

[5] Sinclair, A.N., Fortin, J., Shakibi, B., Honarvar, F., Jastrzebski, M., Moles, M.D.C., Enhancement of ultrasonic images for sizing of defects by time-of-flight diffraction, NDT\&E International, 43 (2010) 258-264.

[6] Petcher, P.A., Dixon, S., A modified Hough transform for removal of direct and reflected surface waves from Bscans, NDT\&E International, 44 (2011) 139-144.

[7] Petcher, P.A., Dixon, S., Parabola detection using matched filtering for ultrasound B-scans, Ultrasonics, 52 (2012) 138-144.

[8] Merazi-Meksen, T., Boudraa, M., Boudraa, B., Mathematical morphology for TOFD image analysis and automatic crack detection, Ultrasonics, 54 (2014) 1642-1648.

[9] Silk, M. G., The transfer of ultrasonic energy in the diffraction technique for crack sizing. Ultrasonics, 17 (1979) $113-$ 121.

[10] Nath, S.K., Balasubramaniam, K., Krishnamurthy, C.V., Narayana, B.H., Reliability assessment of manual ultrasonic time of flight diffraction (TOFD) inspection for complex geometry components, NDT\&E International 43 (2010) 152-162.

[11] Nalbone, L., Adelfio, R., D’arienzo, M., Ingrassia, T., Nigrelli, V., Zabbara, F., Paladini, P., Campi, F., Pellegrini, A., Porcellini, G., Optimal positioning of the humeral component in the reverse shoulder prosthesis, Musculoskeletal surgery, 98(2) (2014) 135-142.

[12] Nath, S. K., Effect of variation in signal amplitude and transit time on reliability analysis of ultrasonic time of flight diffraction characterization of vertical and inclined cracks, Ultrasonics 54 (2014) 938-952.

[13] Ferrand, A., Darmon, M., Chatillon, S., Deschamps, M., Modeling of ray paths of head waves on irregular interfaces in TOFD inspection for NDE, Ultrasonics 54 (2014) 1851-1860.

[14] Krautkramer, J., Krautkramer, H., Ultrasonic Testing of Materials, Springer-Verlag Berlin Heidelberg GmbH, New York, (1977). 